\title{
Mori Contractions of Maximal Length
}

\author{
by
}

Andreas HöRING and Carla NovelLi

\begin{abstract}
We prove a relative version of the theorem of Cho, Miyaoka and Shepherd-Barron: a Mori fibre space of maximal length is birational to a projective bundle.

2010 Mathematics Subject Classification: Primary 14E30; Secondary 14D06, 14J40, 14J45. Keywords: Mori contraction, length of extremal rays, rational curves, degenerations of projective spaces.
\end{abstract}

\section{$\S 1$. Introduction}

\section{§1.A. Motivation}

Let $X$ be a Fano manifold of dimension $d$ with Picard number one, and denote by $H$ the ample generator of the Picard group. Let $i(X) \in \mathbb{N}$ be such that $-K_{X} \equiv$ $i(X) H$; by a classical theorem of Kobayashi and Ochiai [KO73] one has $i(X) \leq d+1$ and equality holds if and only if $X$ is isomorphic to the projective space $\mathbb{P}^{d}$. If one tries to understand Fano manifolds of higher Picard number, the index $i(X)$ is less useful, since it can be equal to one even for very simple manifolds like $\mathbb{P}^{d} \times \mathbb{P}^{d+1}$. For these manifolds the pseudoindex

$$
\min \left\{-K_{X} \cdot C \mid C \subset X \text { a rational curve }\right\}
$$

yields much more precise results, the most well-known being the theorem of Cho, Miyaoka and Shepherd-Barron:

Communicated by S. Mukai. Received January 31, 2012. Revised April 13, 2012, July 6, 2012, and July 27, 2012.

A. Höring: Université Pierre et Marie Curie, Institut de mathématiques de Jussieu, Projet Topologie et géométrie algébriques, Case 247, 4 place Jussieu, 75005 Paris, France; e-mail: hoering@math.jussieu.fr

C. Novelli: Dipartimento di Matematica, Università degli Studi di Padova, via Trieste 63, I-35121 Padova, Italy; e-mail: novelli@math.unipd.it 
Theorem 1.1 ([CMSB02, Cor. 0.3] [Keb02, Thm. 1.1]). Let $X$ be a projective manifold of dimension $d$ such that for every curve $C \subset X$, we have $-K_{X} \cdot C \geq d+1$. Then $X$ is isomorphic to the projective space $\mathbb{P}^{d}$.

Fano manifolds (or more generally Fano varieties with certain singularities) are important objects since they appear naturally in the minimal model program as the general fibres of Mori fibre spaces. However if one wants to get a more complete picture of Mori fibre spaces, one should also try to obtain some information on the special fibres. In the polarised setting we have a relative version of the KobayashiOchiai theorem:

Theorem 1.2 ([Fuj87, Lemma 2.12], [Ion86]). Let $X$ be a manifold and $\varphi: X \rightarrow Y$ a projective, equidimensional morphism of relative dimension $d$ onto a normal variety. Suppose that the general fibre $F$ is isomorphic to $\mathbb{P}^{d}$ and that there exists a $\varphi$-ample Cartier divisor $A$ on $X$ such that the restriction to $F$ is isomorphic to the hyperplane divisor $H$. Then $\varphi$ is the projectivisation of a vector bundle and $A$ is a global hyperplane divisor.

The existence of the $\varphi$-ample Cartier divisor $A$ being a rather restrictive condition, the object of this paper is to replace it by a more flexible "numerical" hypothesis, i.e. we prove a relative version of Cho-Miyaoka-Shepherd-Barron theorem, even for fibrations that are not equidimensional.

\section{$\S 1 . B$. Main results}

Let $X$ be a quasi-projective manifold and let $\varphi: X \rightarrow Y$ be an elementary contraction, i.e. a Mori contraction associated with an extremal ray $R$ of $X$. The length of the extremal ray $R$ (or of the elementary contraction) is defined as

$$
l(R):=\min \left\{-K_{X} \cdot C \mid C \subset X \text { a rational curve such that }[C] \in R\right\} .
$$

Denote by $E \subset X$ an irreducible component of the $\varphi$-exceptional locus $(E=X$ for a contraction of fibre type), and let $F$ be an irreducible component of any $\varphi$-fibre contained in $E$. Then by the Ionescu-Wiśniewski inequality [Ion86, Thm. 0.4], [Wiś91a, Thm. 1.1] one has

$$
\operatorname{dim} E+\operatorname{dim} F \geq \operatorname{dim} X+l(R)-1 .
$$

In this paper we investigate the case when (1.1) is an equality: examples of such a situation are given by projective bundles, i.e. smooth fibrations $\varphi: X \rightarrow Y$ that are locally trivial in the analytic topology with fibre $F \simeq \mathbb{P}^{d}$. If the contraction $\varphi$ maps $X$ onto a point, Theorem 1.1 says that $X=E=F$ is a projective 
space; more generally Andreatta and Wiśniewski conjectured that $F$ is a projective space regardless of the dimension of the target [AW97, Conj. 2.6]. We prove a strong version of this conjecture for Mori fibre spaces:

Theorem 1.3. Let $X$ be a quasi-projective manifold that admits an elementary contraction $\varphi: X \rightarrow Y$ of fibre type onto a normal variety $Y$ such that the general fibre has dimension d. Suppose that the contraction has length $l(R)=d+1$. If $\varphi$ is equidimensional, it is a projective bundle. If $\varphi$ is not equidimensional, there exists a commutative diagram

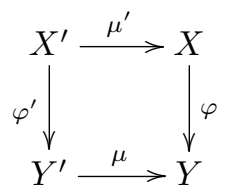

such that $\mu$ and $\mu^{\prime}$ are birational, $X^{\prime}$ and $Y^{\prime}$ are smooth, and $\varphi^{\prime}: X^{\prime} \rightarrow Y^{\prime}$ is a projective bundle.

Since $X$ is smooth, Theorem 1.1 immediately implies that a general $\varphi$-fibre is isomorphic to $\mathbb{P}^{d}$. Our contribution is to prove that the condition on the length severely limits the possible degenerations of these projective spaces. The smoothness of $X$ is not essential for these degeneration results (cf. Section 3), so one can easily derive analogues of Theorem 1.3 making some assumption on the singularities of the general fibre (e.g. isolated LCIQ singularities [CT07]).

If the contraction $\varphi$ is birational, the situation is more complicated:

Theorem 1.4. Let $X$ be a quasi-projective manifold that admits a birational elementary contraction $\varphi: X \rightarrow Y$. Let $E \subset X$ be an irreducible component of the exceptional locus such that for a general fibre of $E \rightarrow \varphi(E)=: Z$ the inequality (1.1) is an equality. Let $\tilde{E} \rightarrow E$ be the normalisation of $E$ and $\tilde{\varphi}: \tilde{E} \rightarrow \tilde{Z}$ be the fibration obtained by the Stein factorisation of $\tilde{E} \rightarrow E \rightarrow Z$. Then $\tilde{\varphi}$ is a projective bundle in codimension one. Moreover, there exists a commutative diagram

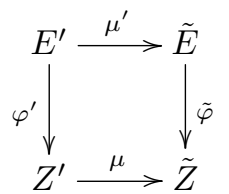

such that $\mu$ and $\mu^{\prime}$ are birational, $E^{\prime}$ and $Z^{\prime}$ are smooth, and $\varphi^{\prime}: E^{\prime} \rightarrow Z^{\prime}$ is a projective bundle.

As in the case of a Mori fibre space, it is not hard to see that the general fibre of $\tilde{\varphi}: \tilde{E} \rightarrow \tilde{Z}$ is a projective space ([CMSB02, Rem. 12], cf. Lemma 2.2), 
so the key point is to study its degenerations. While this result gives a rather precise description of the normalisation $\tilde{E}$ and the fibration $\tilde{\varphi}$, it does not prove that an irreducible component of a general fibre of $\left.\varphi\right|_{E}: E \rightarrow Z$ is a projective space. However, if the contraction $\varphi$ is divisorial (so the inequality (1.1) simplifies to $\operatorname{dim} F \geq l(R)$, Andreatta and Occhetta [AO02, Thm. 5.1] proved that all the nontrivial fibres of $\varphi$ have dimension $l(R)$ if and only if $X$ is the blow-up of a manifold along a submanifold of codimension $l(R)+1$. If one studies the proof of Kawamata's classification of smooth fourfold flips [Kaw89, Thm. 1.1] (which corresponds to the case $\operatorname{dim} X=4, \operatorname{dim} E=\operatorname{dim} F=2, l(R)=1$ ) one sees that for flipping contractions the proof of the normality requires completely different techniques. We leave this interesting problem for future research.

\section{§1.C. Further developments}

Theorem 1.3 completely determines the equidimensional fibre type contractions of maximal length: they are projective bundles. If the fibration is not equidimensional it still gives a precise description of the Chow family defined by the fibration. It seems reasonable to expect that this description will allow one to deduce some information about higher-dimensional fibres. For example if $X$ of dimension $n$ maps onto a threefold $Y$, it is not hard to see that any fibre component of dimension $n-2$ is normalised by $\mathbb{P}^{n-2}$. More generally we expect that the theory of varieties covered by high-dimensional linear spaces [Ein85], [Wiś91b], [BSW92], [ABW92], [Sat97], [NO11] can be applied in this context.

Another interesting line of investigation would be to prove that under additional assumptions the fibration $\varphi$ is always equidimensional. We recall the following conjecture by Beltrametti and Sommese:

Conjecture 1.5 ([BS93], [BS95, Conj. 14.1.10]). Let $(X, L)$ be a polarised projective manifold of dimension $n$ that is an adjunction theoretic scroll $\varphi: X \rightarrow Y$ over a normal variety $Y$ of dimension $m$. If $n \geq 2 m-1$, then $\varphi$ is equidimensional.

Wiśniewski [Wiś91b, Thm. 2.6] proved this conjecture if $L$ is very ample and $n \geq 2 m$, but apart from partial results for low-dimensional $Y$, [BSW92], [Som86], [Tir10], this conjecture is very much open. We expect that Conjecture 1.5 even holds in the more general setting of Theorem 1.3.

Conjecture 1.6. Let $X$ be a projective manifold of dimension $n$ that admits an elementary contraction $\varphi: X \rightarrow Y$ of fibre type onto a normal variety $Y$ of dimension $m$. Suppose that the contraction has length $l(R)=n-m+1$. If $n \geq 2 m-1$, then $\varphi$ is equidimensional. 


\section{$\S 2$. Notation and basic results}

We work over the complex field $\mathbb{C}$. A fibration is a projective surjective morphism $\varphi: X \rightarrow Y$ with connected fibres between normal varieties such that $\operatorname{dim} X>$ $\operatorname{dim} Y$. The $\varphi$-equidimensional (resp. $\varphi$-smooth) locus is the largest Zariski open subset $Y^{*} \subset Y$ such that for every $y \in Y^{*}$, the fibre $\varphi^{-1}(y)$ has dimension $\operatorname{dim} X-$ $\operatorname{dim} Y$ (resp. has $\operatorname{dimension} \operatorname{dim} X-\operatorname{dim} Y$ and is smooth).

An elementary Mori contraction of a quasi-projective manifold $X$ is a morphism $\varphi: X \rightarrow Y$ with connected fibres onto a normal variety $Y$ such that the anticanonical divisor $-K_{X}$ is $\varphi$-ample and the numerical classes of curves contracted by $\varphi$ lie on an extremal ray $R \subset N_{1}(X)$.

We will use the notation of [Kol96, Ch.II]: if $U \rightarrow V$ is a variety $U$ that is projective over some base $V$, we denote by $\operatorname{RatCurves}^{\mathrm{n}}(U / V)$ the space parameterising rational curves on $U$. If moreover $s: V \rightarrow U$ is a section, we denote by $\operatorname{RatCurves}^{\mathrm{n}}(s, U / V)$ the space parameterising rational curves passing through $s(V)$. In the case where $V$ is a point and $x=s(V)$, we simply write $\operatorname{RatCurves}^{\mathrm{n}}(U)$ and RatCurves ${ }^{\mathrm{n}}(x, U)$.

Let $F$ be a normal, projective variety of dimension $d$. If $\mathcal{H} \subset \operatorname{RatCurves}^{\mathrm{n}}(F)$ is an irreducible component parameterising a family of rational curves that dominates $F$, then for a general point $x \in F$ one has

$$
\operatorname{dim} \mathcal{H}_{x}=\operatorname{dim} \mathcal{H}+1-d,
$$

where $\mathcal{H}_{x} \subset$ RatCurves $^{\mathrm{n}}(x, F)$ parameterises the members of $\mathcal{H}$ passing through $x$. If $\mathcal{H}_{x}$ is proper, it follows from bend-and-break [Mor79] that $\operatorname{dim} \mathcal{H}_{x} \leq d-1$. Thus in this case we have

$$
\operatorname{dim} \mathcal{H} \leq 2 d-2
$$

Fix now an ample $\mathbb{Q}$-Cartier divisor $A$ on $F$. Let $C \subset F$ be a rational curve such that

$$
A \cdot C=\min \left\{A \cdot C^{\prime} \mid C^{\prime} \subset F \text { is a rational curve }\right\} ;
$$

then any irreducible component of $\mathcal{H} \subset \operatorname{RatCurves}^{\mathrm{n}}(F)$ containing $C$ is proper [Kol96, Ch. II, Prop. 2.14], so it parameterises a family $\mathcal{H}$ of rational curves that is unsplit in the sense of [CMSB02, Def. 0.2]. If moreover the irreducible component $\mathcal{H}$ has the maximal dimension $2 d-2$, the family is doubly dominant, i.e. for every $x, y \in F$ there exists a member of the family $\mathcal{H}$ that joins $x$ and $y$. In this setting one can prove a version of Theorem 1.1 that includes normal varieties:

Theorem 2.1 ([CMSB02, Main Thm. 0.1]). Let $F$ be a normal, projective variety of dimension $d$ and $A$ an ample $\mathbb{Q}$-Cartier divisor on $F$. Suppose that there exists 
an irreducible component $\mathcal{H} \subset$ RatCurves $^{\mathrm{n}}(F)$ of dimension at least $2 d-2$ such that for a curve $[C] \in \mathcal{H}$ we have

$$
A \cdot C=\min \left\{A \cdot C^{\prime} \mid C^{\prime} \subset F \text { is a rational curve }\right\} .
$$

Then $F$ is isomorphic to a projective space $\mathbb{P}^{d}$ and $\mathcal{H}$ parameterises the family of lines on $\mathbb{P}^{d}$.

Remark. A proof of this statement following the strategy in [Keb02] can be found in $[\mathrm{CT} 07, \S 4]$.

If $F$ is not smooth, it is in general quite hard to verify the condition $\operatorname{dim} \mathcal{H} \geq$ $2 d-2$ (cf. [CT07]). Since we work with an ambient space that is smooth, things are much simpler:

Lemma 2.2. Let $X$ be a quasi-projective manifold that admits a proper birational morphism $\varphi: X \rightarrow Y$ such that $-K_{X}$ is $\varphi$-ample. Set

$$
l(\varphi):=\min \left\{-K_{X} \cdot C \mid C \subset X \text { a rational curve such that } \varphi(C)=\mathrm{pt}\right\} .
$$

Let $E \subset X$ be an irreducible component of the exceptional locus such that

$$
2 \operatorname{dim} E-\operatorname{dim} \varphi(E)=\operatorname{dim} X+l(\varphi)-1 .
$$

Let $F$ be an irreducible component of a general fibre of $E \rightarrow \varphi(E)$. Then the normalisation of $F$ is a projective space.

Proof. If $\varphi(E)$ has positive dimension, let $H \subset Y$ be a general hyperplane section and $X_{H}:=\varphi^{-1}(H)$ its preimage. By adjunction the intersection numbers of $-K_{X}$ and $-K_{X_{H}}$ with the $\varphi$-exceptional curves agree, so we have $l\left(\left.\varphi\right|_{X_{H}}\right) \geq l(\varphi)$. Let $E_{H}$ be an irreducible component of $E \cap X_{H}$ that surjects onto an irreducible component of $\varphi(E) \cap H$. Applying the inequality (1.1) to the birational morphism ${ }^{1}$ $\left.\varphi\right|_{X_{H}}: X_{H} \rightarrow H$ and $E_{H}$ we get

$$
2 \operatorname{dim} E_{H}-\operatorname{dim} \varphi\left(E_{H}\right) \geq \operatorname{dim} X_{H}+l\left(\left.\varphi\right|_{X_{H}}\right)-1 .
$$

Since $\operatorname{dim} E_{H}=\operatorname{dim} E-1$ and $\operatorname{dim} \varphi\left(E_{H}\right)=\operatorname{dim} \varphi(E)-1$ the equality (2.2) implies that this inequality is an equality and $l\left(\left.\varphi\right|_{X_{H}}\right)=l(\varphi)$.

Thus we can take general hyperplane sections on $Y$ until $F$ is an irreducible component of $E$, and the equality (2.2) simplifies to

$$
2 \operatorname{dim} F=\operatorname{dim} X+l(\varphi)-1 .
$$

\footnotetext{
${ }^{1}$ The proof of [Wiś91a, Thm. 1.1] does not use that the contraction is elementary.
} 
Let $C \subset F \subset X$ be a rational curve passing through a general point $x \in F$ that has minimal degree with respect to $-K_{X}$. Then $C$ belongs to an irreducible family $\mathcal{H} \subset$ RatCurves $^{\mathrm{n}}(F)$ of rational curves that dominates $F$ and moreover $\mathcal{H}_{x}$ is proper. Note now that any deformation of $C$ in $X$ is contracted by $\varphi$, hence contained in $F$. Thus we can estimate $\operatorname{dim} \mathcal{H}$ by applying the standard RiemannRoch estimate [CMSB02, p. 14] for the dimension of deformation spaces on the manifold $X$ :

$$
\operatorname{dim} \mathcal{H} \geq-K_{X} \cdot C-3+\operatorname{dim} X \geq l(\varphi)-3+\operatorname{dim} X=2 \operatorname{dim} F-2 .
$$

By (2.1) we see that these inequalities are in fact equalities, in particular one has $-K_{X} \cdot C=l(\varphi)$. Thus if $\nu: \tilde{F} \rightarrow F$ is the normalisation and $\tilde{\mathcal{H}} \subset \operatorname{RatCurves}^{\mathrm{n}}(\tilde{F})$ the family obtained by lifting the members of $\mathcal{H}$, it satisfies the conditions of Theorem 2.1 with respect to the polarisation $A:=-\nu^{*} K_{X}$. Consequently, we have $\tilde{F} \simeq \mathbb{P}^{\operatorname{dim} F}$ and $-\nu^{*} K_{X} \equiv l(R) H$ with $H$ the hyperplane divisor. ${ }^{2}$

\section{§3. Degenerations of $\mathbb{P}^{d}$}

In this section we prove our main results on degenerations of $\mathbb{P}^{d}$ satisfying a length condition.

Proposition 3.1. Let $X$ be a normal, quasi-projective variety and let $\varphi: X \rightarrow Y$ be an equidimensional fibration of relative dimension d onto a normal variety such that the general fibre $F$ is isomorphic to $\mathbb{P}^{d}$. Let $A$ be a $\varphi$-ample $\mathbb{Q}$-Cartier divisor, and let $e \in \mathbb{N}$ be such that $\left.A\right|_{F} \equiv e H$ with $H$ the hyperplane divisor. Suppose that the following length condition holds:

$$
A \cdot C \geq e \quad \forall C \subset X \text { rational curve such that } \varphi(C)=\mathrm{pt} .
$$

Then all the fibres are irreducible and generically reduced. Moreover the normalisation of any fibre is a projective space.

Remark 3.2. In general the degeneration behaviour of projective spaces can be quite complicated, for example it depends in a subtle manner on the geometry of the total space. Consider $\varphi: X \rightarrow C$ a fibration from a normal variety $X$ onto a smooth curve $C$ such that all the fibres are integral and the general fibre is isomorphic to $\mathbb{P}^{d}$. If $X$ is smooth (or at least factorial), then Tsen's theorem implies that $X \rightarrow C$ is a $\mathbb{P}^{d}$-bundle ([NO07, Lemma 2.17], cf. also the proof of [DP10, Lemma 4]). This assumption cannot be weakened:

\footnotetext{
${ }^{2}$ Cf. [Zha91] for an argument assuming smoothness of the general fibre.
} 
Example 3.3 ([AR12]). Let $W_{4} \subset \mathbb{P}^{6}$ be the cone over the Veronese surface $R_{4} \subset \mathbb{P}^{5}$ (that is the 2-uple embedding of $\mathbb{P}^{2}$ in $\mathbb{P}^{5}$ ). The blow-up of $W_{4}$ in the vertex $P$ is isomorphic to the projectivised bundle $\mathbb{P}\left(\mathcal{O}_{\mathbb{P}^{2}} \oplus \mathcal{O}_{\mathbb{P}^{2}}(-2)\right) \rightarrow \mathbb{P}^{2}$; this desingularisation contracts a $\mathbb{P}^{2}$ with normal bundle $\mathcal{O}_{\mathbb{P}^{2}}(-2)$ onto the vertex $P$. In particular $W_{4}$ is a terminal $\mathbb{Q}$-factorial threefold and the canonical divisor $K_{W_{4}}$ is not Gorenstein, but 2-Gorenstein.

The base locus of a general pencil of hyperplane sections of $W_{4} \subset \mathbb{P}^{6}$ identifies to a smooth quartic curve $C \subset R_{4}$. If we denote by $\mu: X \rightarrow W_{4}$ the blow-up in $C$, it is a terminal, $\mathbb{Q}$-factorial, 2-Gorenstein threefold admitting a fibration $\varphi: X \rightarrow \mathbb{P}^{1}$ whose fibres are isomorphic to the members of the general pencil, in particular they are integral. Thus the general fibre $F$ is isomorphic to $R_{4} \simeq \mathbb{P}^{2}$, but the fibre $F_{0}$ corresponding to the hyperplane section through the vertex $P$ is a cone over the quartic curve $C$. In particular it is not normalised by $\mathbb{P}^{2}$.

Let us note that $A:=K_{X} \otimes \mu^{*} \mathcal{O}_{W_{4}}(2)$ is a $\varphi$-ample $\mathbb{Q}$-Cartier divisor such that the restriction to a general fibre is numerically equivalent to the hyperplane divisor $H \subset \mathbb{P}^{2}$. However this divisor is not Cartier, so Theorem 1.2 does not apply. Let us also note that under the 2-uple embedding $\mathbb{P}^{2} \hookrightarrow \mathbb{P}^{5}$, a line is mapped onto a conic. If we degenerate the general fibre $F$ to $F_{0}$, these conics degenerate to a union of two lines $l_{1} \cup l_{2}$ passing through the vertex of the cone $F_{0}$. Since we have $A \cdot l_{i}=\frac{1}{2}$, the length condition (3.1) is not satisfied.

Proof of Proposition 3.1. By assumption the general fibre is a projective space. Let $\mathcal{H} \subset \operatorname{RatCurves}^{\mathrm{n}}(X / Y)$ be the unique irreducible component such that a general point corresponds to a line $l$ contained in the general fibre $F$. We have $A \cdot l=e$ and by (3.1) one has $A \cdot C \geq e$ for every rational curve $C$ contained in a fibre, so the variety $\mathcal{H}$ is proper over the base $Y$ [Kol96, Ch. II, Prop. 2.14]. Since the general fibre of $\mathcal{H} \rightarrow Y$ corresponds to the $2 d-2$-dimensional family of lines in the projective space $\mathbb{P}^{d} \simeq F$, it follows by upper semicontinuity that for every $0 \in Y$, all the irreducible components of the fibre $\mathcal{H}_{0}$ have dimension at least $2 d-2$. Let $\mathcal{H}_{0, i}$ be the normalisation of such an irreducible component and $\mathcal{U}_{0, i} \rightarrow \mathcal{H}_{0, i}$ be the universal family over it. The image of the evaluation morphism $p: \mathcal{U}_{0, i} \rightarrow X$ is an irreducible component $D_{i}$ of the set-theoretical fibre $\left(\varphi^{-1}(0)\right)_{\text {red }}$, so it has dimension $d$. Let $\nu: \tilde{D}_{i} \rightarrow D_{i}$ be the normalisation; then the family of rational curves $\mathcal{H}_{0, i}$ lifts to $\tilde{D}_{i}$, so RatCurves ${ }^{\mathrm{n}}\left(\tilde{D}_{i}\right)$ has an irreducible component of dimension at least $2 d-2$. Since for any rational curve $C \subset D_{i} \subset X$ we have $A \cdot C \geq e$ by (3.1), the pull-back $\nu^{*} A$ also satisfies this inequality. Moreover $\nu^{*} A$ has degree exactly $e$ on the rational curves parameterised by $\mathcal{H}_{0, i}$. Thus we conclude with Theorem 2.1 that $\tilde{D}_{i}$ is isomorphic to a projective space. 
We now suppose for contradiction that there exists a $0 \in Y$ such that the fibre $F_{0}:=\varphi^{-1}(0)$ is reducible or not generically reduced. Then we can decompose the cycle $\left[F_{0}\right]$ as

$$
\left[F_{0}\right]=\sum_{i} a_{i}\left[D_{i}\right]
$$

with $a_{i} \in \mathbb{N}$ and $D_{i}$ the irreducible components of the set-theoretical fibre $\left(\varphi^{-1}(0)\right)_{\text {red }}$. Since the degree is constant in a well-defined family of proper algebraic cycles [Kol96, Prop. 3.12], we have

$$
e^{d}=[F] \cdot A^{d}=\left[F_{0}\right] \cdot A^{d}=\sum_{i} a_{i}\left(\left[D_{i}\right] \cdot A^{d}\right) .
$$

By assumption the sum on the right hand side is not trivial, so $\left[D_{1}\right] \cdot A^{d}<e^{d}$. Denote by $\nu: \mathbb{P}^{d} \rightarrow D_{1}$ the normalisation; then

$$
\left(\nu^{*} A\right)^{d}=\left[D_{i}\right] \cdot A^{d}<e^{d} .
$$

Thus we see that $\nu^{*} A \equiv b H$ with $0<b<e$. Yet this is impossible, since it implies that for a general line $l \subset \mathbb{P}^{d}$, we have

$$
A \cdot \nu(l)=\nu^{*} A \cdot l=b<e,
$$

contradicting (3.1).

Lemma 3.4. Let $\varphi: X \rightarrow Y$ be an equidimensional fibration from a normal variety $X$ onto a manifold $Y$. Suppose that $\varphi$ is generically smooth and the general fibre $F$ is a Fano manifold with Picard number one. If $\varphi$ has irreducible and generically reduced fibres, then $-K_{X}$ is $\mathbb{Q}$-Cartier.

Proof. Denote by $H$ the ample generator of $\operatorname{Pic}(F)$ and let $A$ be a $\varphi$-ample Cartier divisor. Set $e \in \mathbb{N}$ such that $\left.A\right|_{F} \equiv e H$, and $d \in \mathbb{N}$ such that $-K_{F} \equiv d H$. The reflexive sheaf $\mathcal{O}_{X}\left(e K_{X}+d A\right)$ is locally free in a neighbourhood of a general fibre $F$ and the restriction $\mathcal{O}_{X}\left(e K_{X}+d A\right) \otimes \mathcal{O}_{F}$ is isomorphic to the structure sheaf $\mathcal{O}_{F}$. Up to replacing $A$ by $A \otimes \varphi^{*} H$ with $H$ a sufficiently ample Cartier divisor on $Y$ we can suppose without loss of generality that

$$
H^{0}\left(X, \mathcal{O}_{X}\left(e K_{X}+d A\right)\right) \simeq H^{0}\left(Y, \varphi_{*} \mathcal{O}_{X}\left(e K_{X}+d A\right)\right) \neq 0 .
$$

Thus we obtain a nonzero morphism

$$
\mathcal{O}_{X}\left(-e K_{X}\right) \rightarrow \mathcal{O}_{X}(d A)
$$

which is an isomorphism on the general fibre $F$. The vanishing locus is thus a Weil divisor $\sum k_{i} D_{i}$ such that for all $i$ we have $\varphi\left(D_{i}\right) \subsetneq Y$. Since $\varphi$ is equidimensional 
and all the fibres are irreducible and generically reduced we see that $D_{i}=\varphi^{*} E_{i}$ with $E_{i}$ a prime divisor on $Y$. Since $Y$ is smooth, the divisor $E_{i}$ is Cartier, hence $\sum k_{i} D_{i}=\sum k_{i} \varphi^{*} E_{i}$ is Cartier. Thus we have an isomorphism

$$
\mathcal{O}_{X}\left(-e K_{X}\right) \simeq \mathcal{O}_{X}\left(d A-\sum k_{i} \varphi^{*} E_{i}\right)
$$

The right hand side is Cartier, so $K_{X}$ is $\mathbb{Q}$-Cartier.

Proposition 3.5. In the situation of Proposition 3.1, suppose that $Y$ is smooth. Then the fibration $\varphi$ is a projective bundle.

Proof. We will proceed by induction on the relative dimension $d$, the case $d=1$ is [Kol96, Ch. II, Thm. 2.8]. Taking general hyperplane sections on $Y$ and arguing by induction on the dimension we can suppose without loss of generality that $\varphi$ has at most finitely many singular fibres. The problem being local, we can suppose that $Y \subset \mathbb{C}^{\operatorname{dim} Y}$ is a polydisc around 0 and $\varphi$ is smooth over $Y \backslash 0$. Since all the fibres are generically reduced, by Proposition 3.1 we can choose a section $s: Y \rightarrow X$ such that $s(0) \subset F_{0 \text {,nons }}$ where $F_{0}:=\varphi^{-1}(0)$.

As in the proof of Proposition 3.1, we denote by $\mathcal{H} \subset \operatorname{RatCurves}^{\mathrm{n}}(X / Y)$ the unique irreducible component such that a general point corresponds to a line $l$ contained in the general fibre $F \simeq \mathbb{P}^{d}$. There exists a unique irreducible component $\mathcal{H}_{s} \subset \operatorname{RatCurves}^{\mathrm{n}}(s, X / Y)$ such that for general $y \in Y$ the lines in $\varphi^{-1}(y) \simeq \mathbb{P}^{d}$ passing through $s(y)$ are parameterised by $\mathcal{H}_{s}$. If we denote by $\psi: \mathcal{H}_{s} \rightarrow Y$ the natural fibration, its general fibre is isomorphic to a projective space $\mathbb{P}^{d-1}$. Let $q: \mathcal{U}_{s} \rightarrow \mathcal{H}_{s}$ be the universal family; then by [Kol96, Ch. II, Cor. 2.12] the fibration $q$ is a $\mathbb{P}^{1}$-bundle. Let $p: \mathcal{U}_{s} \rightarrow X$ be the evaluation morphism; then $p$ is birational since this holds for the restriction to a general $\varphi$-fibre. The variety $X$ being normal, we know by Zariski's main theorem that $p$ has connected fibres. The family of rational curves being unsplit, it follows from bend-and-break that $p$ has finite fibres over $X \backslash s(Y)$. Thus we have an isomorphism

$$
X \backslash s(Y) \simeq \mathcal{U}_{s} \backslash E,
$$

where $E:=\left(p^{-1}(s(Y))\right)_{\text {red }}$. Again by bend-and-break and connectedness of the fibres the algebraic set $E$ is irreducible, so it is a prime divisor with a finite, birational morphism $\left.q\right|_{E}: E \rightarrow \mathcal{H}_{s}$. Since $\mathcal{H}_{s}$ is normal, we see by Zariski's main theorem that $\left.q\right|_{E}$ is an isomorphism and $E$ is a $q$-section.

By Lemma 3.4 we know that $-K_{X}$ is $\mathbb{Q}$-Cartier, so $-K_{\left(\mathcal{U}_{s} \backslash E\right)}$ is $\mathbb{Q}$-Cartier. Since $\mathcal{U}_{s} \backslash E \rightarrow \mathcal{H}_{s}$ is locally trivial (it is a $\mathbb{C}$-bundle), it follows that $-K_{\mathcal{H}_{s}}$ is $\mathbb{Q}$-Cartier. Since $\mathcal{U}_{s} \rightarrow \mathcal{H}_{s}$ is a $\mathbb{P}^{1}$-bundle, it now follows that $-K_{\mathcal{U}_{s}}$ is $\mathbb{Q}$-Cartier. The $\mathbb{P}^{1}$-bundle $\mathcal{U}_{s} \rightarrow \mathcal{H}_{s}$ has a section $E$, so it is isomorphic to the projectivised 
bundle $\mathbb{P}(V) \rightarrow \mathcal{H}_{s}$ with $V:=\varphi_{*} \mathcal{O}_{X}(E)$. By the canonical bundle formula we have

$$
K_{\mathcal{U}_{s}} \equiv q^{*}\left(K_{\mathcal{H}_{s}}+\operatorname{det} V\right)-2 E .
$$

We claim that the following length condition holds:

$$
-K_{\mathcal{H}_{s}} \cdot C \geq d \quad \forall C \subset \mathcal{H}_{s} \text { rational curve such that } \psi(C)=\text { pt. }
$$

Assuming this for the time being, let us see how to conclude: applying the induction hypothesis to $\psi$, we see that $\mathcal{H}_{s} \rightarrow Y$ is a $\mathbb{P}^{d-1}$-bundle. Since $q$ is a $\mathbb{P}^{1}$-bundle, we see that the central fibre of $\mathcal{U}_{s} \stackrel{\psi \circ q}{\longrightarrow} Y$ is a $\mathbb{P}^{1}$-bundle over $\mathbb{P}^{d-1}$ that contracts a section onto a smooth point of $F_{0}$. A classical argument [Mor79] shows that $F_{0} \simeq \mathbb{P}^{d}$.

Proof of the claim. The claim is obvious for curves in the general fibres which are isomorphic to $\mathbb{P}^{d-1}$, so we can concentrate on curves in the central fibre $\mathcal{H}_{0}:=$ $\left(\psi^{-1}(0)\right)_{\text {red }}$. We set $\mathcal{U}_{0}:=q^{-1}\left(\mathcal{H}_{0}\right)$ and denote by $\nu: \tilde{\mathcal{H}}_{0} \rightarrow \mathcal{H}_{0}$ the normalisation. Then $\mathcal{U}_{0} \times{ }_{\mathcal{H}_{0}} \tilde{\mathcal{H}}_{0}$ is normal and $\tilde{q}_{0}: \mathcal{U}_{0} \times \mathcal{H}_{0} \tilde{\mathcal{H}}_{0} \rightarrow \tilde{\mathcal{H}}_{0}$ is a $\mathbb{P}^{1}$-bundle. By Proposition 3.1 we already know that $F_{0 \text {,red }}$ is normalised by $\mathbb{P}^{d}$, so we obtain a commutative diagram

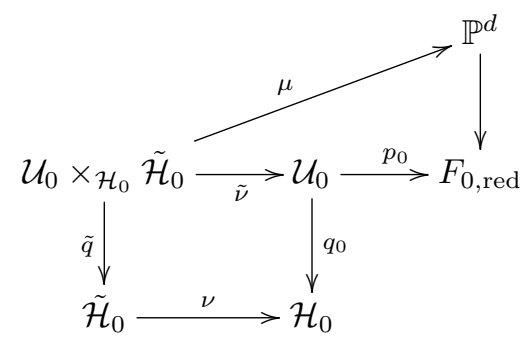

Thus the curves parameterised by $\tilde{\mathcal{H}}_{0}$ are lines in $\mathbb{P}^{d}$, in particular $\tilde{\mathcal{H}}_{0} \simeq \mathbb{P}^{d-1}$ and

$$
\mathcal{U}_{0} \times_{\mathcal{H}_{0}} \tilde{\mathcal{H}}_{0} \simeq \mathbb{P}\left(\mathcal{O}_{\mathbb{P}^{d-1}} \oplus \mathcal{O}_{\mathbb{P}^{d-1}}(-1)\right)
$$

with $\tilde{\nu}^{-1}\left(E \cap \mathcal{U}_{0}\right)$ corresponding to the exceptional section.

In order to check the length condition (3.4) it is sufficient to do this for a curve $C_{0} \subset \mathcal{H}_{0}$ such that $C_{0}=\nu\left(l_{0}\right)$ with $l_{0}$ a general line in $\tilde{\mathcal{H}}_{0} \simeq \mathbb{P}^{d-1}$. We can lift $l_{0}$ to a subsection $l^{\prime} \subset \mathbb{P}\left(\mathcal{O}_{\mathbb{P}^{d-1}} \oplus \mathcal{O}_{\mathbb{P}^{d-1}}(-1)\right)$ that is disjoint from the exceptional section, so we obtain a curve $C^{\prime}:=\tilde{\nu}\left(l^{\prime}\right) \subset \mathcal{U}_{0} \backslash E$ such that $C^{\prime} \rightarrow C_{0}$ is birational. In particular we have

$$
-K_{\mathcal{H}_{s}} \cdot C_{0}=-q^{*} K_{\mathcal{H}_{s}} \cdot C^{\prime},
$$

so it is sufficient to show that the right hand side is equal to $d$. The rational curve $C^{\prime}$ does not meet $E$ and $\mu\left(l^{\prime}\right)$ is a line, so $C^{\prime}$ corresponds to a point in $\mathcal{H}$ that is not 
in $\mathcal{H}_{s}$. Using the isomorphism (3.2) we can deform $C^{\prime}$ to a curve $C^{\prime \prime} \subset \mathcal{U}_{s}$ that is a line in a general fibre $\varphi^{-1}(y) \simeq \mathbb{P}^{d}$ and which does not meet the point $s(y)$, so $C^{\prime \prime}$ is disjoint from $E$. Yet for such a curve the formula (3.3) restricted to a general fibre immediately shows that $-q^{*} K_{\mathcal{H}_{s}} \cdot C^{\prime \prime}=d$.

Remark 3.6. Proposition 3.5 should be true without the assumption that $Y$ is smooth. In fact this assumption is only needed to ensure via Lemma 3.4 that $-K_{X}$ (and by consequence $-K_{\mathcal{H}_{s}}$ and $-K_{\mathcal{U}_{s}}$ ) is $\mathbb{Q}$-Cartier. However our computations only use that these varieties are "relatively $\mathbb{Q}$-Cartier", i.e. some multiple of the canonical divisor is linearly equivalent to a Cartier divisor plus some Weil divisors that are pull-backs from the base $Y$. We leave the technical details to the interested reader. (Note added in proof: this statement has now been proved by Araujo and Druel [AD12, Prop. 4.10].)

\section{$\S 4$. Proofs of main results}

Proof of Theorem 1.3. By Theorem 1.1 the general $\varphi$-fibre is a projective space.

If $\varphi$ is equidimensional, then $A:=-K_{X}$ satisfies the length condition (3.1) in Proposition 3.1 with $e=l(R)$. Thus we know that every fibre is generically reduced. In particular for every $y \in Y$ there exists a point $x \in \varphi^{-1}(y)$ such that the map between the Zariski tangent spaces

$$
T_{\varphi}: T_{X, x} \rightarrow T_{Y, y}
$$

has rank equal to $\operatorname{dim} Y$. Since $X$ is smooth, this implies that $Y$ is smooth at $y$. Conclude with Proposition 3.5.

Suppose now that $\varphi$ is not equidimensional. Let now $\bar{Y}$ be the closure of the $\varphi$-equidimensional locus in the relative Chow variety $\operatorname{Chow}(X / Y)$ and let $\bar{X} \rightarrow \bar{Y}$ be the universal family. Let $Y^{\prime} \rightarrow \bar{Y}$ be a desingularisation, and write $X^{\prime}$ for the normalisation of $\bar{X} \times_{\bar{Y}} Y^{\prime}$. We denote by $\varphi^{\prime}: X^{\prime} \rightarrow Y^{\prime}$ the natural fibre space structure and by $\mu^{\prime}: X^{\prime} \rightarrow X$ the birational morphism induced by the map $\bar{X} \rightarrow X$. By the rigidity lemma there exists a birational morphism $\mu: Y^{\prime} \rightarrow Y$ such that $\mu \circ \varphi^{\prime}=\varphi \circ \mu^{\prime}$. Note also that the restriction of $\mu^{\prime}$ to any $\varphi^{\prime}$-fibre is finite, so the pull-back $A^{\prime}:=-\left(\mu^{\prime}\right)^{*} K_{X}$ is a $\varphi^{\prime}$-ample Cartier divisor. The divisor $A^{\prime}$ on $X^{\prime}$ satisfies the length condition (3.1) in Proposition 3.1 with $e=l(R)$. Since $Y^{\prime}$ is smooth, we can conclude with Proposition 3.5.

Proof of Theorem 1.4. By Lemma 2.2 the general $\tilde{\varphi}$-fibre is a projective space. Moreover if $\nu: \tilde{E} \rightarrow E \subset X$ denotes the normalisation, then $A:=-\nu^{*} K_{X}$ (restricted to the $\tilde{\varphi}$-equidimensional locus) satisfies the length condition (3.1) in Proposition 3.1 with $e=l(R)$. If $C \subset \tilde{Z}$ is a curve cut out by general hyperplane 
sections, then $C$ is smooth and the fibration $\tilde{\varphi}^{-1}(C) \rightarrow C$ is equidimensional, so it is a projective bundle by Proposition 3.5. Thus $\tilde{\varphi}$ is a projective bundle in codimension one.

The proof of the second statement is analogous to the proof of the second statement in Theorem 1.3.

\section{Acknowledgements}

We thank C. Araujo for sending us her inspiring preprint [AR12]. We thank M. Andreatta, S. Druel, H. Hamm, F. Han, P. Popescu-Pampu, M. Wolff and J. A. Wiśniewski for helpful communications.

A. Höring was partially supported by the A.N.R. project "CLASS".

\section{References}

[ABW92] M. Andreatta, E. Ballico and J. A. Wiśniewski, Projective manifolds containing large linear subspaces, in Classification of irregular varieties (Trento, 1990), Lecture Notes in Math. 1515, Springer, Berlin, 1992, 1-11. Zbl 0787.14034 MR 1180333

[AO02] M. Andreatta and G. Occhetta, Special rays in the Mori cone of a projective variety. Nagoya Math. J. 168 (2002), 127-137. Z Zb 1055.14015 MR 1942399

[AW97] M. Andreatta and J. A. Wiśniewski, A view on contractions of higher-dimensional varieties, in Algebraic geometry-Santa Cruz 1995, Proc. Sympos. Pure Math. 62, Amer. Math. Soc., Providence, RI, 1997, 153-183. Zbl 0948.14014 MR 1492522

[AD12] C. Araujo and S. Druel, On codimension 1 del Pezzo foliations on varieties with mild singularities, arXiv:1210.4013, 2012.

[AR12] C. Araujo and J. Ramón-Marí, Flat deformations of $\mathbb{P}^{n}$, arXiv:1212.3593, 2012.

[BS93] M. C. Beltrametti and A. J. Sommese, Comparing the classical and the adjunctiontheoretic definition of scrolls, in Geometry of complex projective varieties (Cetraro, 1990), Sem. Conf. 9, Mediterranean, Rende, 1993, 55-74. Zbl 0937.14027 MR 1225588

[BS95] _ The adjunction theory of complex projective varieties, de Gruyter Expositions Math. 16, de Gruyter, Berlin, 1995. Zbl 0845.14003 MR 1318687

[BSW92] M. C. Beltrametti, A. J. Sommese and J. A. Wiśniewski, Results on varieties with many lines and their applications to adjunction theory, in Complex algebraic varieties (Bayreuth, 1990), Lecture Notes in Math. 1507, Springer, Berlin, 1992, 16-38. Zbl 0777.14012 MR 1178717

[CT07] J.-C. Chen and H.-H. Tseng, Note on characterization of projective spaces, Comm. Algebra 35 (2007), 3808-3819. Zbl 1135.14041 MR 2362685

[CMSB02] K. Cho, Y. Miyaoka, and N. I. Shepherd-Barron, Characterizations of projective space and applications to complex symplectic manifolds, in Higher dimensional birational geometry (Kyoto, 1997), Adv. Stud. Pure Math. 35, Math. Soc. Japan, Tokyo, 2002, 1-88. Zbl 1063.14065 MR 1929792

[DP10] S. Druel and M. Paris, Characterizations of projective spaces and hyperquadrics, Asian J. Math., to appear; arXiv:1012.5238, 2010.

[Ein85] L. Ein, Varieties with small dual varieties. II, Duke Math. J. 52 (1985), 895-907. Zbl 0603.14026 MR 0816391 
[Fuj87] T. Fujita, On polarized manifolds whose adjoint bundles are not semipositive, in Algebraic geometry (Sendai, 1985), Adv. Stud. Pure Math. 10, North-Holland, Amsterdam, 1987, 167-178. Zbl 0659.14002 MR 0946238

[Ion86] P. Ionescu, Generalized adjunction and applications, Math. Proc. Cambridge Philos. Soc. 99 (1986), 457-472. Zbl 0619.14004 MR 0830359

[Kaw89] Y. Kawamata, Small contractions of four-dimensional algebraic manifolds, Math. Ann. 284 (1989), 595-600. Zbl 0661.14009 MR 1006374

[Keb02] S. Kebekus, Characterizing the projective space after Cho, Miyaoka and ShepherdBarron, in Complex geometry (Göttingen, 2000), Springer, Berlin, 2002, 147-155. Zbl 1046.14028 MR 1922103

[KO73] S. Kobayashi and T. Ochiai, Characterizations of complex projective spaces and hyperquadrics, J. Math. Kyoto Univ. 13 (1973), 31-47. Zbl 0261.32013 MR 0316745

[Kol96] J. Kollár, Rational curves on algebraic varieties, Ergeb. Math. Grenzgeb. 32, Springer, Berlin, 1996. MR 1440180

[Mor79] S. Mori, Projective manifolds with ample tangent bundles, Ann. of Math. (2) 110 (1979), 593-606. Zbl 0423.14006 MR 0554387

[NO07] C. Novelli and G. Occhetta, Ruled Fano fivefolds of index two. Indiana Univ. Math. J. 56 (2007), 207-241. Zbl 1118.14048 MR 2305935

[NO11] bundle, Michigan Math. J. 60 (2011), 441-462. Zbl 1229.14015 MR 2825270

[Sat97] E. Sato, Projective manifolds swept out by large-dimensional linear spaces, Tohoku Math. J. (2) 49 (1997), 299-321. Zbl 0917.14026 MR 1464179

[Som86] A. J. Sommese, On the adjunction theoretic structure of projective varieties, in Complex analysis and algebraic geometry (Göttingen, 1985), Lecture Notes in Math. 1194, Springer, Berlin, 1986, 175-213. Zbl 0601.14029 MR 0855885

[Tir10] A. L. Tironi, Scrolls over four dimensional varieties, Adv. Geom. 10 (2010), 145-159. Zbl 1194.14079 MR 2603726

[Wiś91a] J. A. Wiśniewski, On contractions of extremal rays of Fano manifolds, J. Reine Angew. Math. 417 (1991), 141-157. Zbl 0721.14023 MR 1103910

[Wiś91b] On deformation of nef values, Duke Math. J. 64 (1991), 325-332. Zbl 0773.14003 MR 1136378

[Zha91] Q. Zhang, Extremal rays on higher-dimensional projective varieties, Math. Ann. 291 (1991), 497-504. Zbl 0762.14009 MR 1133347 